\title{
OH MEGAMASER PUMPING MODELS
}

\author{
N. D. KYLAFIS AND K. G. PAVLAKIS \\ University of Crete \\ Physics Department \\ P.O. Box 2208 \\ 71003 Heraklion, Crete, Greece \\ kylafis@physics.uch.gr \\ pavlakis@ast.leeds.ac.uk
}

\section{Introduction}

We would like to review the current status of $\mathrm{OH}$ megamaser pumping models. Contrary to the quantity and quality of observations of $\mathrm{OH}$ megamasers, surprisingly little theoretical modeling has been done on them so far.

After the discovery of the first OH megamaser in Arp220 (Baan, Wood, \& Haschick 1982), and with the discovery of several others, a revealing correlation was found (Martin et al. 1988; Baan 1989), namely

$$
L_{\mathrm{OH}} \propto L_{\mathrm{FIR}}^{2},
$$

where $L_{\mathrm{OH}}$ is the $\mathrm{OH}$ megamaser luminosity in the $1667 \mathrm{MHz}$ line and $L_{\mathrm{FIR}}$ is the far infrared (FIR) luminosity of the host galaxy as measured by IRAS.

A tighter correlation was found by Baan et al. (1992), namely

$$
L_{\mathrm{OH}} \propto L_{60 \mu \mathrm{m}}^{2},
$$

where $L_{60 \mu \mathrm{m}}$ is the $60 \mu \mathrm{m}$ luminosity of the host galaxy. In either case, such a correlation hints that the pumping of the OH molecules is done by the FIR radiation. Below we will consider other pumping mechanisms also.

One can understand the above correlations as follows: It is well known (see, e.g., Elitzur 1982) that the FIR radiation can, in principle, invert the populations of $\mathrm{OH}$. (Recently we have had an observational verification of this by Skinner et al. 1997.) Thus, the inverted populations of $\mathrm{OH}$ amplify the background radio contimuum at $18 \mathrm{~cm}$, and under the assumption of weak amplification it is expected that

$$
L_{\mathrm{OH}} \propto L_{\mathrm{FIR}} L_{\text {radio }}
$$

However, for the nuclear components of active galaxies it has been shown (Norris, Allen, \& Roche 1988) that

$$
L_{\text {radio }} \propto L_{\mathrm{FIR}}
$$

and therefore relation (1) is understood.

\section{Characteristics of the $\mathrm{OH}$ Megamaser Clumps}

From our knowledge so far, the typical $\mathrm{OH}$ megamaser chump has the following characteristics:

The number density of $\mathbf{H}_{2}$ molecules is

$$
n_{11_{2}} \lesssim \text { few } \times 10^{4} \mathrm{~cm}^{-3},
$$

and the fractional abundance of $\mathrm{OH}$ molecules is

$$
\begin{gathered}
n_{\mathrm{OH}} / n_{\mathrm{H}_{2}} \sim 10^{-6}\left(\text { possibly } 10^{-5}\right) . \\
946
\end{gathered}
$$

J. Andersen (ed.), Highlights of Astronomy, Volume 11B, 946-948.

(C) 1998 IAU. Printed in the Netherlands. 
The color temperature of the FIR radiation emitted by the dust is

$$
T_{\text {dust }} \sim 60 \mathrm{~K}
$$

and the gas temperature is also

$$
T_{\text {gas }} \sim 60 \mathrm{~K} .
$$

The typical size of a megamaser clump is

$$
d_{\text {compact }} \sim 1 \mathrm{pc}
$$

for the compact clumps, while for the diffuse emission the size is

$$
d_{\text {diffuse }} \sim 100 \mathrm{pc} .
$$

Finally, large velocities (of order hundreds to a few thousand $\mathrm{km} \mathrm{s}^{-1}$ ) are seen in the spectra, but it is not clear yet whether there are systematic velocity gradients in the emitting medium.

\section{Pumping Mechanisms of $\mathbf{O H}$}

Of all possible pumping mechanisms of the $\mathrm{OH}$ molecules, three are the main ones for the conditions prevailing in $\mathrm{OH}$ megamasers:

1. Collisions between $\mathrm{OH}$ and $\mathrm{H}_{2}$. Collisional rate coefficients were recently calculated by Offer, van Hemert, \& van Dishoeck (1994).

2. Spectral line overlap. For clarity of presentation we will distinguish two types of line overlap, though both types occur at the same time and cannot be separated. We will say that two spectral lines overlap locally if the rest frequencies of the two lines are separated by less than their thermal width. On the other hand, two spectral lines overlap nonlocally if there are velocity gradients in the medium and velocity differences large enough to Doppler shift one line into the other. For a formalism to treat radiation transport in the Large Velocity Gradient (or Sobolev) approximation, with the effects of line overlap included, see Pavlakis \& Kylafis (1996a).

3. Far infrared radiation coming from dust emission. See, e.g., Elitzur (1982) for a review.

\section{Model Calculations}

Not much work has been done so far on model calculations of $\mathrm{OH}$ megamasers. From an extensive search of the literature we have come up with only three papers: Henkel, Güsten, \& Baan (1987), Burdyuzha \& Vikulov (1990), and Randell et al. (1995). This list does not include observational papers, which have some theoretical remarks in them.

Indirectly rolevant to $\mathrm{OH}$ megamasers are the calculations of Pavlakis \& Kylafis $(1996 \mathrm{~b}, \mathrm{c}$ ), which refer to $\mathrm{OH}$ masers in Galactic star-forming regions. At low densities $\left(n_{\mathrm{H}_{2}} \lesssim\right.$ few $\times 10^{4}$ $\left(\mathrm{m}^{-3}\right)$, these calculations hint at what is happening in $\mathrm{OH}$ megamasers.

We will comment on each one of the above four papers in reverse chronological order. For each paper we will give its pluses and minuses.

Pavlakis \& Kylafis (1996c, see their Figure 6) find that at low densities $\left(n_{\mathrm{H}_{2}} \lesssim\right.$ few $\left.\times 10^{4} \mathrm{~cm}^{-3}\right)$ the luminosity of the $1667 \mathrm{MHz}$ line is larger than the luminosity of the $1665 \mathrm{MHz}$ line. This is exactly what is seen in $\mathrm{OH}$ megamasers and it may explain the diffuse $\mathrm{OH}$ maser emission seen in Galactic stiar-forming regions (Mirabel, Rodriguez, \& Ruiz 1989). At higher densities (appropriate for HII regions), the calculations show just the opposite for the 1667 and $1665 \mathrm{MHz}$ lines, in agreement with observations. The parameter space explored by Pavlakis \& Kylafis $(1996 \mathrm{~b}, \mathrm{c})$ is generally not appropriate for $\mathrm{OH}$ megamasers. The calculations must explore lower densities, lower temperatures and larger velocity gradients.

Randell et al. (1995) have done by far the largest number of calculations regarding $\mathrm{OH}$ megamasers. They have included all the pumping mechanisms and have reproduced qualitatively the data for several megamasers. However, their models practically always show the $4765 \mathrm{MHz}$ line as a maser, which is contrary to observations (Henkel, Güsten, \& Baan 1987). Also, despite their efforts, they were unable to invert the problem and from maser (and non-maser) observations to infer physical conditions. 
Burdyuzha \& Vikulov (1990), with FIR radiation from dust and microturbulence, have managed to make all four $(1667,1665,1720$, and $1612 \mathrm{MHz})$ ground state transitions to exhibit inverted populations. However, they always find that the ratio of the $1667 \mathrm{MHz}$ to the $1665 \mathrm{MHz}$ line luminosities is between 1 and 1.2, which is contrary to the observations (e.g., Henkel \& Wilson 1990; Kazes \& Baan 1991). For the excited state lines they give no information.

Henkel, Güsten, \& Baan (1987), with a relatively simple model have managed to capture the essential ingredients of $\mathrm{OH}$ megamaser pumping (i.e., FIR radiation and nonlocal line overlap). They have succeeded in explaining the 100 pc scale maser emission (not the compact components) and they have invoked exponential (not linear) amplification for the 1667 and $1665 \mathrm{MHz}$ lines (i.e., $|\tau| \sim$ few). This is now confirmed for IIIZw35 by Trotter et al. (1997). Since the main purpose of the work of Henkel, Güsten, \& Baan (1987) was to report the discovery of the $4.7 \mathrm{GHz},{ }^{2} \Pi_{1 / 2} J=1 / 2$, rotationally excited $\mathrm{OH}$ lines in megamaser galaxies, they performed only a few model calculations of $\mathrm{OH}$ megamasers.

\section{Summary}

With the recent ISO and VLBA results, and the ones to come in the near future (also by Arecibo), the $\mathrm{OH}$ megamaser problem is ripe for inversion, i.e., to derive diagnostics of physical conditions from line observations. For this, accurate models exploring the parameter space are needed. We (Pavlakis \& Kylafis 1997) are currently computing such models, but other people are encouraged to do the same thing in order to build confidence in the results.

\section{References}

Baan, W. A. 1989, ApJ, 338, 804

Baan, W. A., Rhoads, J., Fisher, K., Altschuler, D. R., \& Haschick, A. 1992, ApJ, 396, L99

Baan, W. A., Wood, P. A. D., \& Haschick, A. D. 1982, ApJ, 260, L49

Burdyuzha, V. V. \& Vikulov, K. A. $1990, M N R A S, \mathbf{2 4 4}, 86$

Elitzur, M. 1982, Rev. Mod. Phys., 54, 1225

Henkel, C., Güsten, R., \& Baan, W. A. 1987, $A \& A, 185,14$

Henkel, C. \& Wilson, T. L. 1990, $A \& A, \mathbf{2 2 9}, 431$

Kazes, I, \& Baan, W. A. 1991, $A \& A, \mathbf{2 4 8}$, L15

Martin, J. M., Bottinelli, L., Dennefeld, M., Gouguenheim, L., \& Le Squeren, A. M. 1988, $A \& A, 201, \mathrm{~L} 13$

Mirabel, I. F., Rodriguez, L. F., \& Ruiz, A. 1989, ApJ, 346, 180

Norris, R. P., Allen, D. A., \& Roche, P. F. 1988, MNRAS, 234, 773

Offer, A. R., van Hemert, M. C. \& van Dishoeck, E. F. 1994, J. Chem. Phys., 100, 362

Pavlakis, K. G. \& Kylafis, N. D. 1996a, ApJ, 467, 292

Pavlakis, K. G. \& Kylafis, N. D. 1996b, $A p J, 467,300$

Pavlakis, K. G. \& Kylafis, N. D. $1996 \mathrm{c}, A p J, 467,309$

Pavlakis, K. G. \& Kylafis, N. D. 1997, in preparation

Randell, J., Field, D., Jones, K. N., Yates, J. A., \& Gray, M. D. 1995, A\& A, 300, 659

Skinner, C. J., Smith, H. A., Sturm, E., Barlow, M. J., Cohen, R. J., \& Stacey, G. J. 1997, Nature, 386, 472

Trotter, A. S., Moran, J. M., Greenhill, L. J., Zheng, X.-W., \& Gwinn, C. R. 1997, ApJ, 485, L79 